\title{
ACO Applied to Switch Engine Scheduling in a Railroad Yard
}

\author{
Jodelson A. Sabino ${ }^{1,2}$, Thomas Stützle ${ }^{2}$, \\ Mauro Birattari ${ }^{2}$, and José Eugênio Leal ${ }^{1}$ \\ 1 Departamento de Engenharia Industrial \\ Pontifícia Universidade Católica do Rio de Janeiro, Rio de Janeiro, Brazil \\ jel@ind.puc-rio.br \\ 2 IRIDIA, CoDE, Université Libre de Bruxelles, Brussels, Belgium \\ jsabino@iridia.ulb.ac.be, \{stuetzle, mbiro\}@ulb.ac.be
}

This reasearch studies ACO algorithms for the switch engine scheduling in a Railroad Yard. The cars are moved individually or grouped into blocks by a set of locomotives called switch engines which are dedicated to moving around the cars in the yard. The need for moving comes from the fact that the arriving trains are disassembled into blocks of cars, undergo some operations like, loading, unloading and cleaning and finally are assembled into a new train. Each moving request is called a switch order. The decision of which switch engine will execute which switch order and the sequence of that executution is the core of our problem. The optimization of this schedule reduces the overall operational costs of the yard as weel as the time to assemble new trains, thus leading to a more productive railroad system.

The problem can be summarized as follows: Given the information about the railroad yard layout, the switch engines currently located in it and a list containing all pending planned switch orders the goal is to determine an assignment of switch engines to switch orders, and a sequencing of these such that none of the operational constraints are violated and the costs are minimized. The practical goal os the overall project is to develop a switch engine scheduling algorithm for the Tubarao Railroad Yard, located in Brazil, which is the largest railroad yard in Latin America.

The switch engine scheduling problem is strongly connected to the multiple pickup and delivery problem with time windows (m-PDPTW), which is used to model passenger and good transportation. This is a well known NP-hard problem from which heuristic approaches have shown to produce solutions that are easy to implement and have a low sensitivity to changes in the original problem. A first contribution of our work is the adaptation of an ACO algorithm, in this case COMPETants [1] to the problem, introducing a number of railroad yard specific constraints to model the particularities of the railroad yard scenario.

First tests using real-world instances showed that our algorithm might produce huge savings. Hence we decided to continue the research with further analysis and possibly improving the original algorithm. For these tests we have deployed an instance generator to produce additional sets of data based on parameters that describe the most important operational characteristics of the yard. In our initial tests, we examined the implementations of two different pheromone 


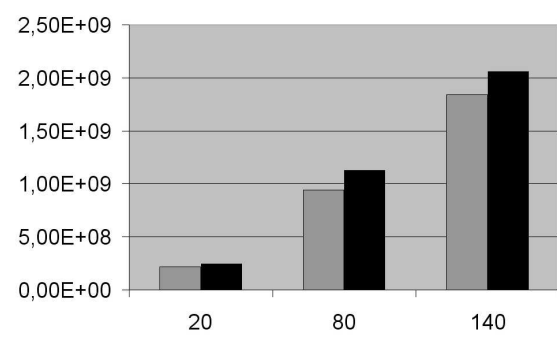

(a) Wide time windows

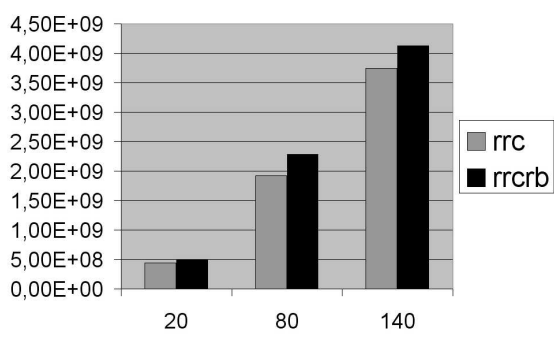

(b) Tight time windows

Fig. 1. RRCRB and RRC solution values comparison for tight and wide time windows, considering plans with 20,80 and 120 switch orders

update rules. The first one, which we called RRC (Railroad COMPETants) was simply the same rule used in the COMPETants algorithm and the second one, called RRCRB (Railroad COMPETants - Rank Based) used the pheromone update rule presented in [2] as the Rank Based Ant System.

An extensive analysis was done comparing the quality of the solutions obtained with both implementations. It showed that the RRC version of the algorithm outperformed the RRCRB version for all the combinations of input parameters and instance type and sizes considered. This can be seen in Figure 1 , which illustrates one the results obtained. Paired-t tests showed that both variations deliver results that vary accordingly up and down. We also concluded that further tuning of the algorithm parameters seems not to be very promising to improve the performance of the worse variant.

As the next steps in this research, we plan to try to improve the solution quality obtained by the ACO algorithm through the adoption of more performing ACO techniques or the inclusion of local search procedures. We also plan to extend the scope of this research tackling other problems in the railroad yard operation algorithmically.

\section{References}

1. Reimann, M.: Ant Based Optimization in Goods Transportation. PhD thesis, University of Vienna (2002)

2. Dorigo, M., Stützle, T.: Ant Colony Optimization. The MIT Press (2004) 